\title{
Methods and Characteristics of Physical Training in College Middle and Long-distance Runners
}

\author{
Fuquan Wang ${ }^{1}$ \\ ${ }^{1}$ Chongqing Real Estate College, China
}

Keywords: colleges and universities; middle and long-distance runners; physical training; methods and characteristics

\begin{abstract}
The middle and long-distance running belongs to the cycle-endurance project of physical fitness type, and the distance is longer, the intensity belongs to the sub-maximum intensity work, to overcome the self-weight, and the same movement is repeated for a long time as a technical feature. At present, with the improvement of competitive level and fierce competition, the requirements for athletes' speed level are getting higher. The physical fitness plays a dominant and core role in the composition of its competitive ability. The ability of middle and long-distance runners to win in a fierce competition depends mainly on the size of their physical fitness.
\end{abstract}

\section{Introduction}

The middle and long-distance running sports refer to a kind of track and field sports of more than 800 meters. At the ancient Greek Olympic Games more than 2,000 years ago, there was a middle-distance running project. In the middle of the 19th century, the middle-distance and long-distance running movements had a very good effect on people's physical fitness, and they began to be popular in some European countries [1]. All countries in the world have also begun to pay attention to middle-distance running. China's sports career started late. In the early 1990s, It held the first middle-distance race competition. With more and more sports events between countries, sports competitions such as middle-distance running are no longer simple. For the purpose of physical exercise, it is also a sign to measure the physical quality of a country's people. To improve the performance of middle-distance running is to increase the speed of players, and how to improve the speed in the training of middle-distance running has also become the focus of many coaches.

\section{Physical Characteristics of Middle and Long-distance Running}

Physical fitness is based on the energy metabolism of the three major energy supply systems of the human body and the exercise capacity demonstrated by the skeletal muscle system. Physical fitness includes physical fitness, form, and function, as shown in Figure 1. Each of the three elements has its own relatively independent role and is closely related to each other. It restricts and influences each other. The level of one of these factors will affect the overall level of physical fitness [1]. Among them, the change of form and function is the material basis of the change of physical ability. The exercise quality is the external manifestation of physical performance. In sports training, the development of various sports qualities is the basic content of physical fitness training.

With the improvement of living standards, people's physical fitness has been greatly improved. Competition for the physical fitness of middle-distance race is also more and more intense. The world record of the middle-distance race competition project is constantly being refreshed and can be seen. In the initial middle-distance race, all players were able to maintain the same speed and decide the outcome in the final stage of sprint [2]. However, with the fierce competition, several steps have already been able to be separated in many middle-distance races, especially in some in the long-distance running project; this shows that the players are no longer simply comparing the final sprinting ability, but also the speed in the middle-distance race competition. It can be seen that the speed competition is not the competition of the sprint; the middle-distance race also starts to speed. The demands are getting higher and higher. 


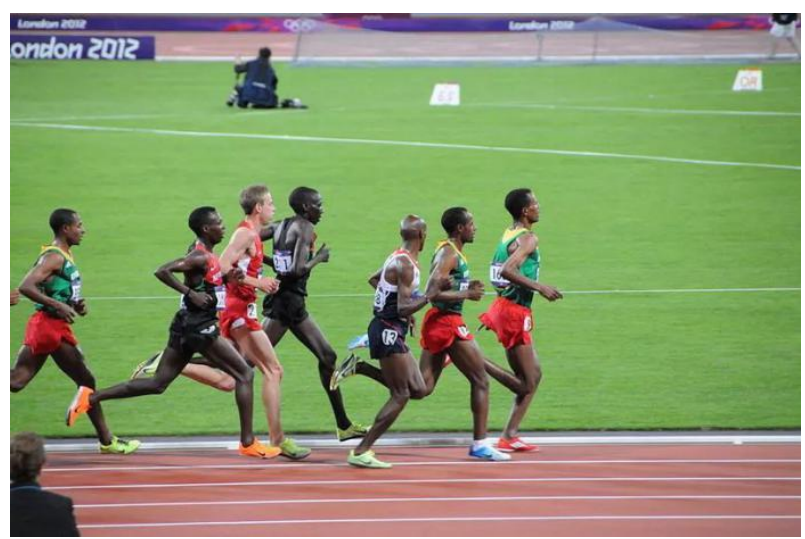

Fig.1 characteristics of middle and long distance running

\section{Physical Training Methods and Means in Middle and Long-distance Running}

Speed endurance is the foundation, speed is the core, speed power and strength endurance are guaranteed. As to whether or not the middle-distance runner can produce results, the athlete's specific goal depends on his $400 \mathrm{~m}$ speed. The $400 \mathrm{~m}$ speed is used as an indicator to evaluate the speed endurance of middle-distance runners.

The specific indicators of athletic quality in the middle run include: standing and jumping, vertical jump, speed $100 \mathrm{~m}, 400 \mathrm{~m}$, power jerk, $1500 \mathrm{~m}$, and flexibility. In training, athletes should pay attention to the balanced development of various physical qualities. Speed is the core, speed endurance is the basis, and strength endurance is the guarantee [2].

The athlete's strength, speed and endurance are likened to the three angles of the triangle that are all in balance with the center point. When raising one corner, the other two corners will be at a lower position, if two of them are raised at the same time. At the corner, the other corner is also at a lower position.

In short, raising three corners at the same time is difficult, but it is necessary. By maintaining a balanced development of strength, speed, and speed endurance, athletes can speed up the overall level of physical fitness.

Although this argument is purely theoretical, it still helps people to understand the special qualities required by middle-distance runners, and has an in-depth understanding of the relationship among strength, speed, and speed endurance. In the training of daily long-distance running, speed training, endurance training and strength training are the key parts of the special training for middle-distance runners.

\subsection{The quality of speed training.}

Speed quality refers to the ability of the body's rapid movement; it is the basis for endurance. The speed quality of middle and long-distance runners is crucial to improving their performance, especially in high-speed running and sprinting. The maximum speed quality is not a determinant factor of the middle-distance running project [3]. Therefore, to increase the speed of the athlete must be coordinated with other factors affecting the improvement of the speed endurance level. Single maximum speed-improving speed training, like a sprinter's speed training, may gain maximum speed, but its speed translates into a small rate of speed endurance.

Middle-distance runners need both non-lactic anaerobic metabolic energy supply training and glycol-tic anaerobic metabolic energy supply training. The simultaneous improvement of these two speeds can promote the athlete's speed endurance level [1]. The development of non-lactate energy speed capability is a special requirement of the endurance project. It is the development of the athlete's maximum speed at the athlete's organism at a certain amount of lactic acid accumulation.

In practice, the coaches generally arrange for maximum speed training when the lactic acid content in the athlete's organism is not restored after the special training load. 


\subsection{Training methods to improve high-speed running ability:}

1) Repeatedly running: using more than $80 \%$ of the intensity, each group repeatedly runs $150 \mathrm{~m}$, 250m, 500m distance 4-5 times, each group rests about 20min;

2) Intermittent run: run $100 \mathrm{~m}$ run with nearly $100 \%$ intensity, then jog for $1 \mathrm{~min}$, intermittent practice;

3) Exhausted and repeated running: Use special game distance or slightly longer distance, run 100\% strength for several times, and fully rest between each time.

Improve the sprint running ability training method, as shown in Figure 2:

1) $3 \times 60 \mathrm{~m}$ large pace running, jogging return at rest;

2) $3 \times 60 \mathrm{~m}$ jogging began to gradually accelerate; the last $10 \mathrm{~m}$ reached the maximum speed, jogging at rest to return;

3) $3 \times 30-60 \mathrm{~m}$ high leg lift running, jogging return at rest;

4) $3 \times 30-60 \mathrm{~m}$ jumping fast run, jogging back at rest;

5) $3 \times 60 \mathrm{~m}$ variable speed running, 20m forward and backward jumping, $20 \mathrm{~m}$ jogging in the middle, jogging return at rest;

6) $3 \times 60 \mathrm{~m}$ starting fast running practice, jogging back at rest.

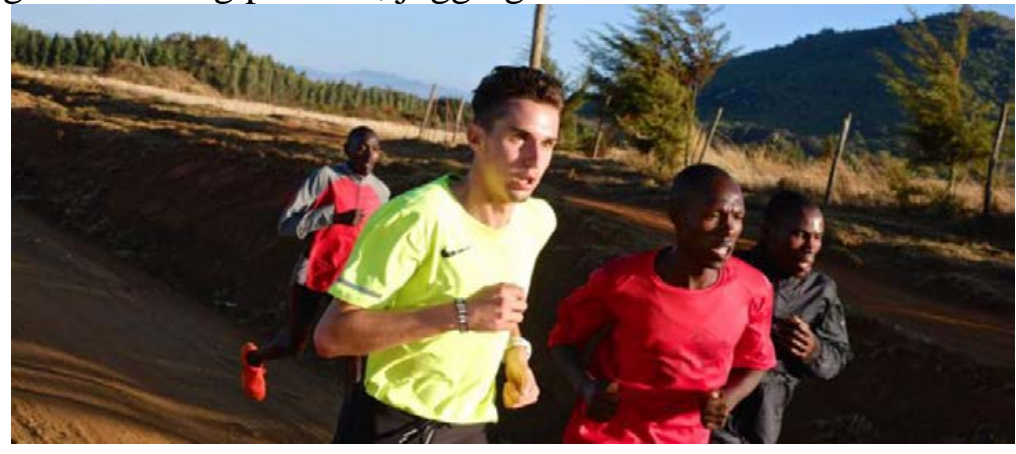

Fig.2 Improve training for sprinting

\subsection{Endurance quality training.}

Endurance quality is the most basic quality of middle and long distance runners.

1) General endurance. General endurance is the synthesis of athletes' organs and organ systems. It is the basis for specific endurance in different projects, as shown in Figure 3. Its task is to systematically influence various factors that affect endurance during the general physical training process, expand the organism's functional capacity for general work, establish conditions for increasing the load, and use the effects of quality transfer to lay the foundation for the development of special endurance [3].

According to the analysis of the characteristics of the middle-distance running project, general endurance training is closely related to the improvement of cardiovascular and respiratory functions. The ability to adapt to strength for a long period of continuous work is "the performance of aerobic endurance." The task of "aerobic endurance": The first is to establish preconditions for improving exercise load. The second is the effect of transferring stamina to a special practice.

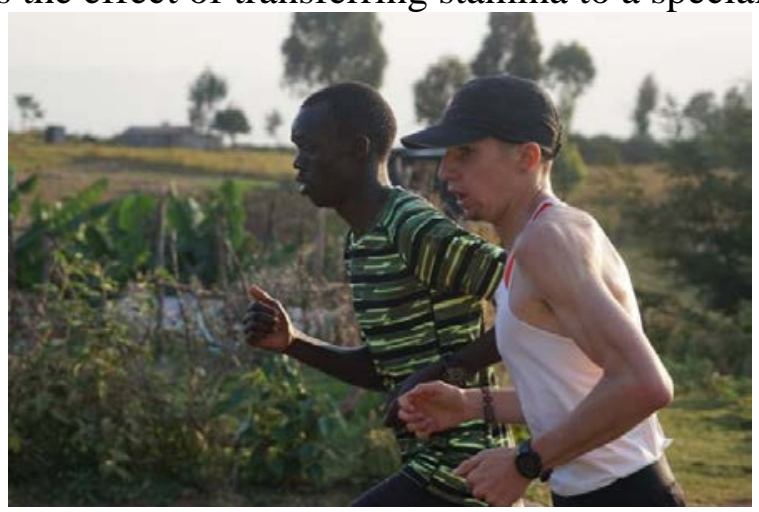

Fig. 3 General endurance training 
2) Special endurance. Special endurance is the ability of the athlete's organism to maximize its ability to mobilize the ability to overcome the fatigue caused by the special load in order to obtain a special performance.

The special endurance feature of the middle and long-distance running project is to pass the entire process with the highest possible average speed. The exercise content is mainly arranged using near-specific exercise distances and exercise intensity. The main methods are: Intermittent training, repetition training and so on.

Lactic acid is the enemy of athletes of the cycle endurance project. From the biological perspective of training, during exercise, lactic acid accumulation leads to body fatigue or functional decline, which is one of the major factors affecting athletic ability [4]. However, a large accumulation of lactic acid can stimulate the body's buffering and adaptation of acidic substances, thereby increasing glycolysis energy supply capacity. The maximum intensity of exercise is between 30 seconds and 15 minutes. It belongs to the mixed energy supply of non-lactic acid and glycolysis, in which glycolysis energy supply plays a dominant or important role in the long-distance running competition.

Therefore, middle-distance runners need both non-lactic acid anaerobic metabolic energy supply training and glycolysis anaerobic metabolic energy supply training. The simultaneous improvement of these two speeds can promote the athlete's speed endurance level.

Develop general endurance training methods:

1) Continuous running at constant speed: Run as much load as possible, and exercise time is above 1h. Heart rate control is around 150 beats/min;

2) Cross-country running: the running speed can be properly changed, the heart rate is controlled at 150-170 times/min, and the exercise time is 1.5-2 hours;

3) Variable speed running: The load intensity is from low to high, the heart rate is controlled at 130-150 times/min, 170-180 times/min, and the exercise duration is more than half an hour;

4) Intermittent running: The training load is small, and the duration of each exercise in the training is not long. When the load intensity is high, the heart rate reaches $170-180$ beats/min. The next exercise is performed with the body not completely recovered, and the heart rate is between 120-140 times/min;

5) Altitude training develops anaerobic endurance training methods: regular intermittent time running, gradual shortening of intermittent time running, short paragraph intermittent running, and long paragraph intermittent running.

6) Aerobic and anaerobic mixed endurance training methods: repeated running, intermittent fast running, exhaustive repeated running, short-distance repeated running, continuous relay.

Of course, according to the specific characteristics of the middle-long distance race and the individual characteristics of the athletes, in different training years and different training periods, different quality endurance training must strictly control different training proportions in order to achieve the goal of improving performance.

\section{Main Methods of Physical Training for College Long-distance Runners}

\subsection{Focus on genetic advantages and adopt progressive training methods.}

When universities and colleges select long-distance runners, they must fully consider the genetic factors of athletes, select athletes with higher talent among athletes, and perform physical training for middle and long-distance running [5]. After the selection, a reasonable training plan is formulated. In the training process, the coaches should formulate a physical training program based on the athlete's superiority, so as to enhance the athlete's long-distance running level. In the physical training process, the coaches must fully understand the main structure of the human body and the choice of function to carry out training methods, and perform top-notch training for athletes with better training performance. The training of middle and long distance runners should follow the principle from simple to difficult [3]. The amount of training should be increased from small to large, and the intensity of training should be gradually enhanced. Finally, high-intensity training should be conducted. 


\subsection{Strengthen theoretical guidance and improve the scientific level of self-training.}

Before the physical training in the middle and long distance races, the coaches must work out a scientific and reasonable training plan. Before that, the coaches must fully master the theoretical knowledge; learn the theoretical content of physical training, as well as the knowledge of sports physiology and theoretical knowledge of sports training [5]. Combining theoretical knowledge with training practice, and regularly learning new knowledge, improving the theoretical knowledge and skills of sports training, combined with the world's cash training technology, in order to formulate the most scientific and most suitable for the training of student athletes [6]. The coaches also need to organize the training content, pass the training techniques to the student's athletes, and coaches and middle-and-long-distance runners must make common progress. The coaches should strengthen communication with the athletes so that the athletes can better understand the training program and help improve the training awareness and autonomy of the middle and long-distance runners.

\subsection{Strengthen special training by using plate training mode.}

In the long-distance running training, it is necessary to change the traditional training pattern and mode, and through the way of plate training, the technical items and physical performance items are refined to form a modular and unitary training model. Combine one or two items into a training module through a fitness project to form two training sections [6]. After the plate training, the intensive training is carried out at the same time, and the conventional training and concentrated intensive training are performed at the same time, so that the strength and complexity of the training are increased, and the training difficulty is continuously enhanced. Dividing the training into different stages, the coaches worked out the time for intensive training, and thus alternated two plate trainings [4]. When the plate is still in a stable state, the new intensive training starts again, and the new intensive training has a higher starting point, and the state of the training will be in a relatively long period of time.

\section{Conclusion}

The main factors that determine the performance of middle-distance and long-distance running are the strength of speed, speed, and speed endurance. They are the three abilities that must be balanced developed. If the athlete only focuses on the development of speed and endurance, it will be faced with the unsynchronized development of speed and strength. The risk of injury can lead to the lack of oxygen in the muscles, which can cause the athletes to lose elasticity in their muscles and even end their sports career prematurely.

Only after years of systematic training in physical fitness can we reach the peak of the human body's limitless anaerobic metabolic energy supply and sustained high "speed" movement, and create the best sports performance.

\section{References}

[1] H.W. Wang, The impact of the implementation of the new rules on the training arrangements for middle-distance and long-distance running, Beijing University of Sport, 2013, vol.2, pp.7-9.

[2] K.J. Shen, Training Thinking Brought by the Features of Five Running Races, Sport Science Research,2010, vol.2, pp.15-16.

[3] L.F. Zhu and P.J. Chen, Energy consumption measurement method and its application, Chinese Journal of Sports Medicine.2011, vol.6, pp.11-12.

[4] W.Ch. Qian, How to improve speed and conduct special training in middle and long distance running, Sport Science Research,2016, vol.6, pp.21-23.

[5] B.B. Wang, Pediatric nurses and patients to communicate effect, Jilin Medicine. 2010, vol.7, pp.9-10.

[6] H.L. Zhang, Long-distance running physical training: the need for scientific system, Chinese Journal of Sports Medicine, 2014, vol.5, pp.54-56. 